\title{
Recovery of Thyroid Hormone Biosynthesis after a Single Injection of Methylthiouracil as Judged by Thyroidal Radioiodine Uptake, Thyroidal Protein-Bound Iodine, Thyroidal Radioiodinated Amino Acids and Thyroglobulin in the Rat*
}

\author{
YASUO SUGITA, SHIN-ICHI SHIMODA AND KoJIRO SHICHIJO \\ First Department of Internal Medicine, School of Medicine, \\ Gunma University, Maebashi, Japan
}

\begin{abstract}
Synopsis
In an attempt to assess the synthesis of thyroid hormone in the rat after a single large dose of methylthiouracil, radioiodine was injected 0 to $164 \mathrm{hrs}$. after administration of $20 \mathrm{mg}$ methylthiouracil (MTU). Thyroidal radioiodine uptake, thyroidal $\mathrm{PB}^{131} \mathrm{I}$, thyroidal radioiodinated amino acids and thyroidal content of thyroglobulin were measured.

When radioiodine was injected at 0 time, $80 \%$ of total thyroidal radioiodine was in organic form at $24 \mathrm{hrs}$., while thyroidal radioiodine uptake was extremely low because of rapid loss of ${ }^{131} \mathrm{I}^{-}$from the blood in MTU treated animals. Thyroidal PB131 I does reflect MIT but not $\mathrm{T}_{4}$ and $\mathrm{T}_{3}$. On the other hand, when radioiodine was injected $4 \mathrm{hrs}$. before autopsy, all thyroidal radioiodine uptake, thyroidal $\mathrm{PB}^{131} \mathrm{I}_{\mathrm{I}}$ and thyroidal $\mathrm{T}_{4}$ and $\mathrm{T}_{3}$ returned to normal between 3 and 4 days, indicating a good correlation among different thyroid parameters. DIT/MIT ratio, however, showed some delay in its return. Thyroidal content of thyroglobulin decreased when thyroid hormone synthesis was blocked by MTU, but returned to normal when synthesis of thyroid hormone was normal. It is suggested that methylthiouracil acts on the thyroid in two ways; one, blocking oxydative enzyme and the other, depressing thyroglobulin content.
\end{abstract}

It has been established for some time that antithyroid drugs of the thianamide series interfere with synthesis of thyroid hormones, primarily by inhibiting both the oxidation of inorganic iodide and its subsequent incorporation into tyrosyl radicales (Greer et al., 1964). In addition to blockade of oxidation of iodide, Roche (Roche, 1951) have reported a decrease of thyroidal content of thyroglobulin after a long-term administration of antithyroid drug. Blocking action of antithyroid drugs on thyroid hormone synthesis is more complicated, since it

Received for publication November 7, 1968. *Dedicated to Professor Yosoji ITO in commemoration of his unselfish devotion for over fifteen years to the advancement of endocrinological science through the editorial management of Endocrinologia Japonica. has been demonstrated that the smaller quantities of antithyroid drugs than used customarily to induce goiter will preferentially inhibit the diiodination of tyrosine and coupling of iodinated tyrosine radicales to form thyroxine and triiodothyronine, while monoiodination of tyrosine actually may be increased as the production of other iodinated amino acids is being reduced (Slingerland et al., 1959; Richards and Ingbar, 1959; Iino et al., 1961). Thus, when a single large dose of antithyroid drug is administered, a number of parameters of the thyroid function may initially and uniformly be affected in a large measure, because of a marked depression of thyroid hormone synthesis. However, when amounts of circulating antithyroid drug decrease progressively with time, discrepancies in thyroid parameters may 
be developed, because of partial block of thyroid hormone synthesis. Such a sequence of hypothetical events has not been studied in detail in man and experimental animals, however.

The present experiment is designed to study such conditions by administering a single large dose of methylthiouracil and by measuring thyroidal radioiodine uptake, thyroidal protein-bound iodine and thyroidal radioiodinated amino acids in the rat. Attempt is also made to study whether or not thyroidal content of thyroglobulin is affected by a single large dose of methylthiouracil.

\section{Materials and Methods}

One hundred and seventy two adult male Wistar rats, weighing approximately $200 \mathrm{~g}$, were used in this experiment. Animals were fed with a pellet diet* and water ad lib. Tracer dose (5 to $20 \mu \mathrm{c}$ ) of carrier free radioiodine $\left(\mathrm{Na}^{1}{ }^{31} \mathrm{I}\right)$ were injected intraperitoneally 0 to $164 \mathrm{hrs}$. after a single injection of $20 \mathrm{mg}$ methylthiouracil (MTU). The animals were sacrificed 4 to 48 hrs. after injection of radioiodine. The thyroid was cleanly dissected and thyroidal radioactivity was determined using a scintillation counter. The thyroids were homogenized in a glass homogenizer and hydrolyzed with $0.5 \mathrm{ml}$ of pronase solution $(10 \mathrm{mg}$ pronase $/ 10 \mathrm{mg}$ thyroid weight) at $\mathrm{pH} 7.4$ at $37^{\circ} \mathrm{C}$ for 12 hrs. Portions of the digests were subjected to ascending paper chromatography in butanol-acetic acid-water $(4: 1: 5)$ and butanol-ethanol $0.5 \mathrm{~N}$ ammonium $(5: 1: 2)$. For the analysis of thyroidal protein bound iodine $\left(\mathrm{PB}^{131} \mathrm{I}\right)$, portions of homogenates were subjected to ascending paper chromatography in 2 solvent systems. Iodide, monoiodotyrosine (MIT), diiodotyrosine (DIT), thyroxine $\left(\mathrm{T}_{4}\right)$ and triiodothyronine $\left(\mathrm{T}_{3}\right)$ were employed as carriers. The chromatographed strips were then put on x-ray film to obtain radioautographs. The strips were then cut into 5 pieces for ${ }^{131} \mathrm{I}^{-}$analysis depending on the radioautogram. The ${ }^{131} \mathrm{I}$ content of the various components was expressed as percentage of the total ${ }^{13}{ }^{1} \mathrm{I}$ found on each strip. No attempt was made to calculate absolute amounts of intrathyroidal iodinated amino acids. Strips with carrier iodide, MIT, DIT, $\mathrm{T}_{4}$ and $\mathrm{T}_{3}$

* Iodine content of this diet was approximately 200 $-300 \mu \mathrm{g} 127 \mathrm{I}^{-} / \mathrm{kg}$. were stained with ceric sulfate arsenious acid reagent. For the analysis of thyroidal proteins, the thyroids were homogenized by adding physiologic saline to obtain at least $0.5 \%$ protein concentration. Portions of these homogenates were subjected to ultracentrifugation as reported previously (Ui and Tarutani, 1961).

\section{Results}

Experiment 1. Effect of a single injection of $20 \mathrm{mg}$ methylthiouracil on thyroidal radioiodine uptake

As an initial step, the thyroidal radioiodine uptake was used to evaluate blocking action of MTU on thyroid hormone synthesis. Ninety animals were divided into 17 equal groups. Animals of 8 groups received $20 \mathrm{mg}$ MTU and radioiodine i.p. at 0 time and were sacrificed 15 mins., 1, 2, 4, 8, 12, 24 and 48 hrs. after MTU administration. Control animals of 9 groups received saline and radioiodine simultaneously and were killed 15 and 30 mins., 1, 2, 4, 8, 12, 24 and $48 \mathrm{hrs}$.

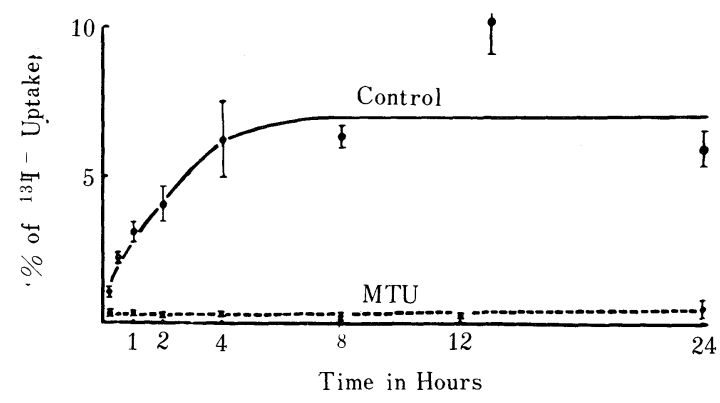

Fig. 1. Effect of a single injection of $20 \mathrm{mg}$ MTU on thyroidal radioiodine uptake. Circles and vertical lines indicate mean $\pm \mathrm{SE}$. Radioiodine and methylthiouracil (MTU) are injected at 0 time.

thereafter. The results were shown in Figure 1. In the control groups, thyroidal radioiodine uptake increased progressively with time until 4 hrs., but was constant thereafter. In sharp contrast to this pattern, thyroidal radioiodine uptake in MTU treated groups was markedly 
depressed throughout $24 \mathrm{hrs}$. at the level of approximately $0.3 \%$.

Since thyroidal radioiodine uptake was governed by thyroidal trapping ability of radioiodine as well as blood concentration of $131 \mathrm{I}^{-}$blood concentration of $131 \mathrm{I}^{-}$was measured in MTU treated groups. As shown in Figure 2, a rapid loss of ${ }^{131} I^{-}$from the blood did occur in MTU treated animals. The ${ }^{1{ }^{11} \mathrm{I}^{-}}$ concentration at $24 \mathrm{hrs}$. was approximately $1 / 8$ to $1 / 10$ of the concentration at $2 \mathrm{hrs}$.

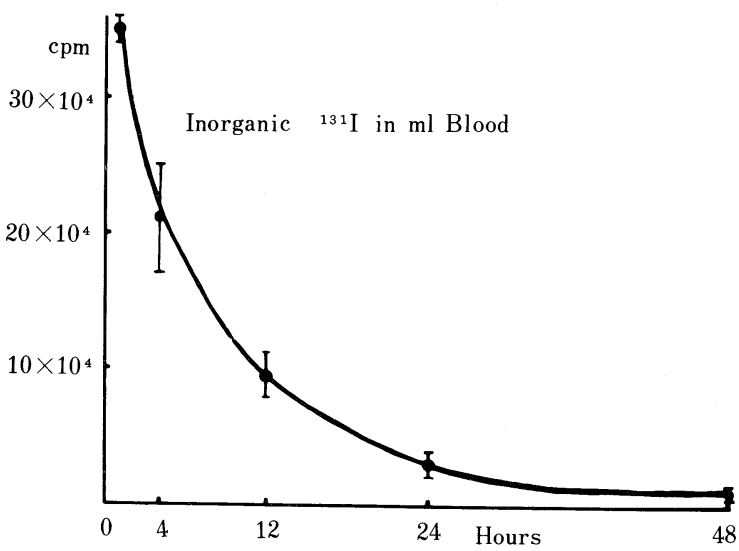

Fig. 2. Disappearance of ${ }^{131} \mathrm{I}$ from the blood in MTU J treated animals. Radioiodine and MTU are injected at 0 time.

Experimetn 2. Effect of a single injection of $20 \mathrm{mg}$ methylthiouracil on thyroidal protein bound iodine

In our next step, blocking action of MTU on thyroid hormone synthesis was assessed by measuring intrathyroidal $\mathrm{PB}^{13}{ }^{1} \mathrm{I}$. The thyroid homogenates were obtained from the animals of experiment 1 , and $\mathrm{PB}^{131} \mathrm{I}$ as the percentage of total radioactivity was measured by paper chromatography. As shown in Figure 3 , more than $95 \%$ of thyroidal radioactivity was $\mathrm{PB}^{131} \mathrm{I}$ as early as 15 mins. and approximately $99 \%$ was $\mathrm{PB}^{131} \mathrm{I}$ later than $8 \mathrm{hrs}$. in the control groups. As expected, a negligible amount of organic iodine was found 15 mins.

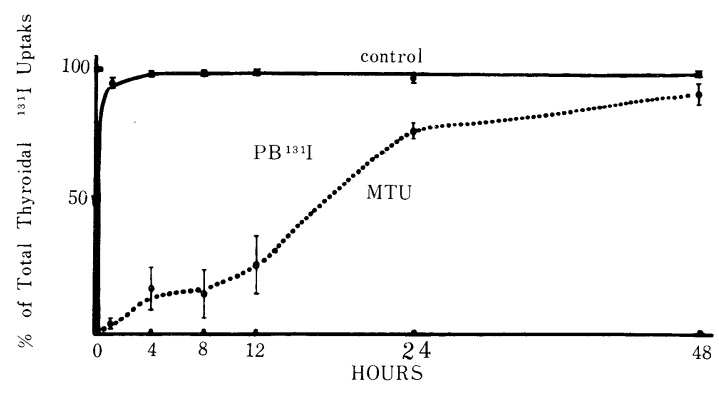

Fig. 3. Thyroidal $\mathrm{PB}^{131} \mathrm{I}$ as the percentage of total thyroidal ${ }^{131} \mathrm{I}$ is indicated. Circles and vertical lines indicate mean $\pm \mathrm{SE}$. Radioiodine and MTU are injected at 0 time.

after MTU and radioiodine administration. However, $\mathrm{PB}^{131} \mathrm{I}$ increased progressively with time and more than $80 \%$ of thyroidal radioiodine was organically bound at $24 \mathrm{hrs}$. in MTU treated animals. A further increase of $\mathrm{PB}^{131} \mathrm{I}$ was found and its value reached approximately normal at $48 \mathrm{hrs}$. in MTU treated animals.

Experiment 3. Effect of a single injection of methylthiouracil on thyroidal radioiodinated amino acids

In our third step, measurement of intrathyroidal radioiodinated amino acids was used to assess blocking action of MTU on thyroid hormone synthesis. The digests of thyroid homogenates were obtained from animals of experiment 1 , and percentage of thyroidal radioiodinated amino acids was analyzed by paper chromatography. As shown in Figure 4, approximately $32 \%$ of total thyroidal radioactivity was MIT at $1 \mathrm{hr}$. in the control animals. The percentage decreased gradually thereafter and reached plateau $(28 \%)$ at 12 hrs. Synthesis of DIT was also rapid and DIT constituted $47 \%$ of total thyroidal radioactivity at $1 \mathrm{hr}$. This percentage was roughly 


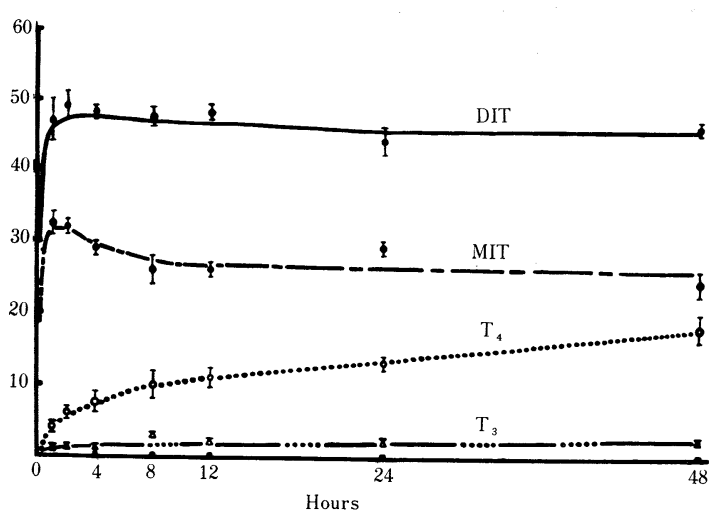

Fig. 4. Changes in intrathyroidal iodinated amino acids in various time intervals after ${ }^{131} \mathrm{I}$ injection in the HID fed rat. Radioactivity of MIT, DIT (diiodotyrosine), T4 (thyroxine), T3 (triiodothyronine) is expressed as per cent of total thyroidal radioactivity. Circles and vertical lines indicate ne. mean \pm SE. Radioiodine and MTU are injected at 0 time.

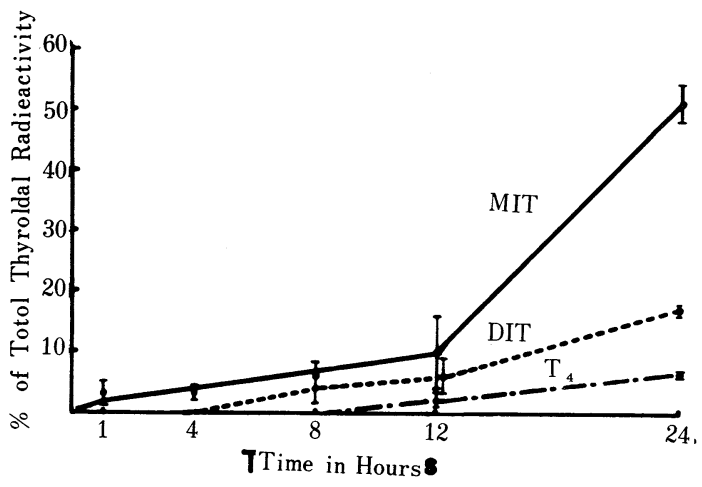

Fig. 5. Formation of thyroidal iodinated amino acid after a single injection $20 \mathrm{mg}$ MTU. Circles and vertical lines indicate mean $\pm S E$. Radioiodine and MTU are injected at 0 time.

constant throughout $48 \mathrm{hrs}$. Synthesis of $\mathrm{T}_{4}$ increased progressively throughout $48 \mathrm{hrs}$. On the other hand, $\mathrm{T}_{3}$ synthesis reached plateau as early as $8 \mathrm{hrs}$.
In sharp contrast to control animals, only a negligible amount of MIT was found $1 \mathrm{hr}$. after MTU and radioiodine administration. No other radioiodinated amino acids were detectable at this time (Fig. 5). This strong inhibition of thyroid hormone synthesis did not continue for extended time, however. For instance, MIT increased gradually with time by $12 \mathrm{hrs}$. and rapidly thereafter. At $24 \mathrm{hrs}$. MIT constituted more than $50 \%$ of thyroidal radioactivity. DIT was detectable at $4 \mathrm{hrs}$. and increased gradually with time. $\mathrm{T}_{4}$ was detectable at $8 \mathrm{hrs}$. and increased similarly with time.

Experiment 4. Effect of a single injection of methylthiouracil on thyroidal radioiodine uptake, thyroidal organic iodine and radioiodinated amino acids when assessed 4 hrs. after radioiodine administration

In our fourth step, thyroidal radioiodine uptake, thyroidal organic iodine and thyroidal radioiodinated amino acids were measured to assess effect of a single injection of methylthiouracil on thyroid hormone synthesis by injecting radioiodine $4 \mathrm{hrs}$. before sacrifice. Eighty two animals were divided into 14 groups. The animals of eleven groups received a single injection of $20 \mathrm{mg}$ MTU at 0 time. Radioiodine was injected at $0,4,8,12,16$, $20,44,68,92,116$, and 164 hrs., and animals

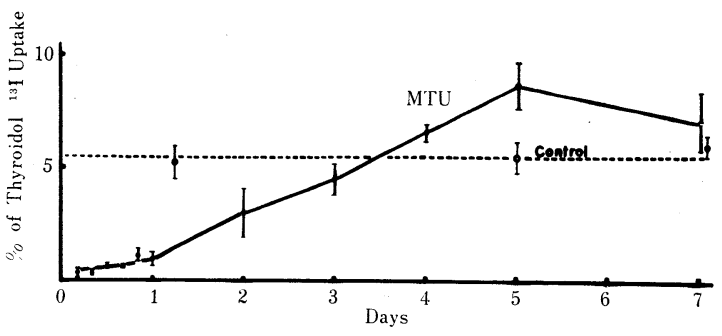

Fig. 6. Thyroidal radioiodine 4 hour uptake after a $20 \mathrm{mg}$ MTU injection. Circles and vertical lines indicate mean $\pm \mathrm{SE}$. Radioiodine is injected $4 \mathrm{hrs}$. before autopsy. 


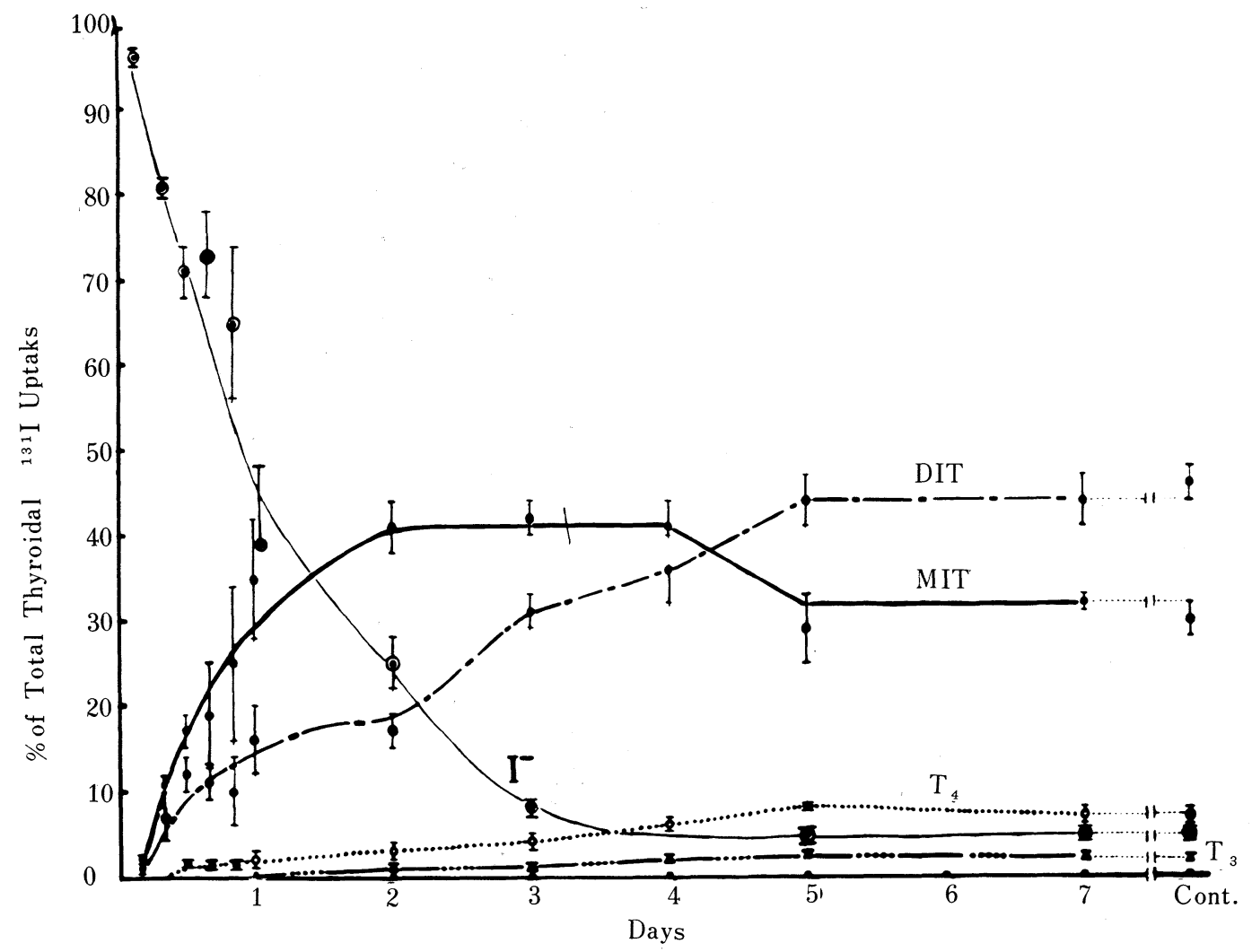

Fig. 7. Changes in intrathyroidal iodinated substances following a single large dose of MTU. Radioiodine is injected $4 \mathrm{hrs}$. before autopsy. Circles and vertical lines indicate mean $\pm \mathrm{SE}$.

were sacrificed 4 hrs. after radioiodine administration. As shown in Figire 6, $4 \mathrm{hrs}$. thyroidal radioiodine uptake was $5 \%$ in the 3 control groups. In MTU treated animals, however, thyroidal radioiodine uptake was markedly low at 4, 8, 12, 16, 20 and 24 hrs. The uptake increased gradually with time thereafter, and reached normal value between 3 and 4 days. Furthermore, the uptake exceeded the control value at 4 and 5 days. The uptake at 7 days was approximately normal, however.

When thyroid homogenate was analysed by paper chromatography, only iodide and original substance $\left(\mathrm{PB}^{131} \mathrm{I}\right)$ were found. When MTU was given, the pattern of intrathyroidal iodide was very similar to that found in Figure 7, and thus alterations of intrathyroidal $\mathrm{PB}^{131} \mathrm{I}$ were not indicated here. However, it should be noted that thyroidal $\mathrm{PB}^{131} \mathrm{I}$ increased with time and reached normal value between 3 and 4 days.

When thyroidal digests were analysed by paper chromatography, more than $95 \%$ of thyroidal radioiodine was iodide $4 \mathrm{hrs}$. after administration of MTU and radioiodine (Fig. 7). As expected, amounts of iodide decreased sharply with time and reached control value between 3 and 4 days. On the other hand, thyroidal radioiodinated amino acids increased progressively with time. The most striking is the rapid increase of MIT, which constituted 
more than $40 \%$ of total thyroidal radioiodine at 2, 3 and 4 days. This percentage of MIT decreased slightly between 4 and 5 days and reached normal value at 5 days. DIT increased less rapidly and reached normal value at 5 days. $\mathrm{T}_{4}$ and $\mathrm{T}_{3}$ increased slowly with time and reached normal value between 3 and 4 days.

As one of the criteria of thyroid hormone synthesis, DIT/MIT ratio was calculated from the data of Figure 7. As shown in Figure 8 , DIT/MIT ratio reached normal value at 5 days.

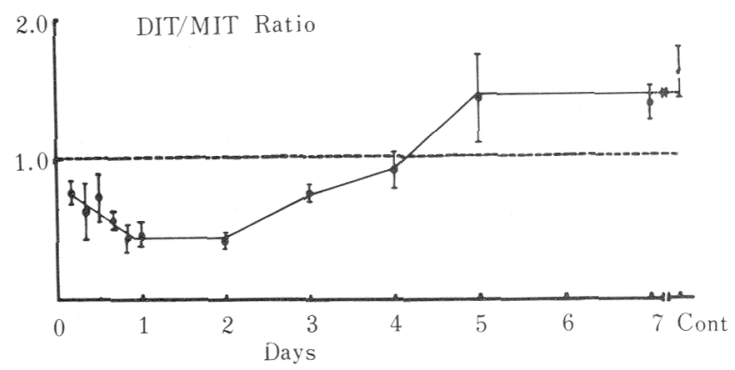

Fig. 8. DIT/MIT ratio. Control value is indicated on the right hand. Circles and vertical lines indicate mean $\pm \mathrm{SE}$.

Experiment 5. Effect of a single injection of methylthiouracil on thyroglobulin

Thyroid proteins were analysed using ultracentrifugation technique. Nine mins. after reaching $50740 \mathrm{rpm}$, the protein patterns were photographed. Figure 9 indicates such protein Patterns in control (A), 4 (B), 24 (C) and 40 (D) hrs. after a single injection of $20 \mathrm{mg}$ methylthiouracil. In the control group, a striking peak of $19 \mathrm{~S}$ thyroglobulin was found. The peak of $19 \mathrm{~S}$ fraction decreased somewhat at $4 \mathrm{hrs}$. The decrease was clearly shown at $24 \mathrm{hrs}$. In addition, $27 \mathrm{~S}$ fraction decreased at $4 \mathrm{hrs}$. and was undetectable thereafter. Interestingly, the peak of $19 \mathrm{~S}$ fraction returned to normal at $40 \mathrm{hrs}$.

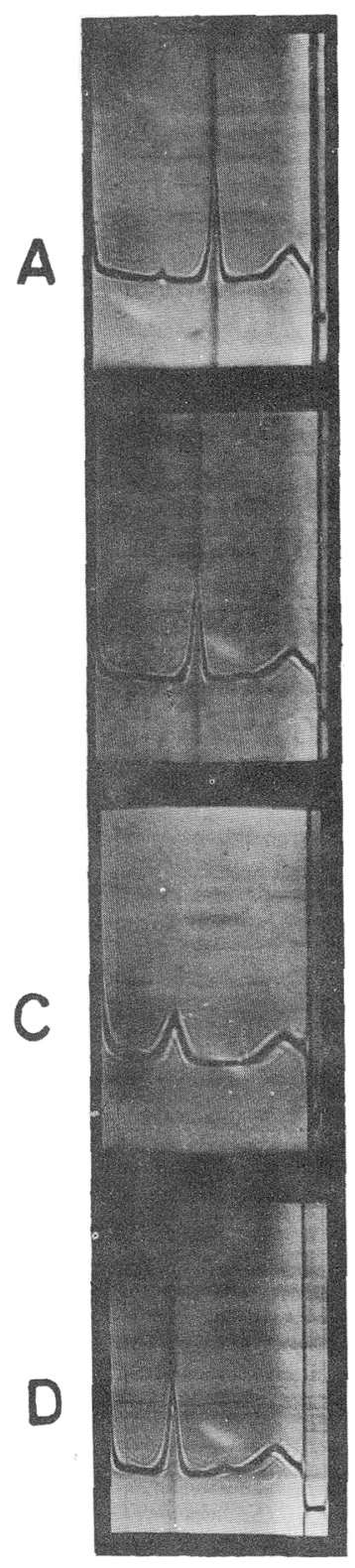

Fig. 9. Ultracentrifugation patterns of thyroidal proteins. A, control group; B, 4 hrs. after MTU; C, 24 hrs. after MTU; D, 40 hrs. after MTU. From left to right, 27S, $19 \mathrm{~S}$ and $4 \mathrm{~S}$. 


\section{Discussion}

A number of criteria have been used to assess blocking action of antithyroid drugs on thyroid hormone synthesis. Since antithyroid durg inhibits synthesis of organic iodine which normally constitutes more than $98 \%$ of thyroidal iodine, it is theoretically possible that administration of antithyroid drug results in a decrease of thyroidal radioiodine uptake (Iino et al. , 1961). In fact, thyroidal radioiodine uptake was low for up to $24 \mathrm{hrs}$. in our present experiment in which $20 \mathrm{mg}$ MTU was administered simultaneously with radioiodine. If this decrease of thyroidal radioiodine uptak accurately reflects the magnitude of blocking action of antithyroid drug on thyroid hormone synthesis, then the measurement of thyroidal radioiodine uptake might be the most simplest technique to assess antithyroid action. However, question arises as to its significance. Since intrathyroidal antithyroid drug can easily be reached out (Shimoda and Greer, 1966), continuation of a low uptake for $24 \mathrm{hrs}$. should indicate that an appreciable amount of antithyroid drug is still circulating at $24 \mathrm{hrs}$. This seems unlikely in view of the fact that disappearance of antithyroid drug from the blood is rapid (Maloof and Soodak, 1957). To solve this discrepancy, we have measured blood ${ }^{131} \mathrm{I}^{-}$in MTU treated animals, since thyroidal radioiodine uptake is governed by both thyroid ability for trapping iodine and amounts of circulating iodide. It is clearly shown that ${ }^{131} \mathrm{I}^{-}$disappeared quite rapidly from the blood and its concentration at 24 hrs. was about $1 / 8$ to $1 / 10$ of initial concentration in MTU treated animals. This agrees well with the report by Brown (Brown, 1956). If iodide trapping ability of the thyroid did not significantly differ between control and MTU treated animals, such a rapid decrease of blood ${ }^{131} \mathrm{I}^{-}$should result in a decrease of thyroidal radioiodine uptake. Therefore, it seems that thyroidal radioiodine uptake does not exactly reflect blooking action of MTU on thyroid hormone synthesis when the uptake is measured long time after administration of radioiodine.

To settle such a question, a direct measurement of organic iodine in the thyroid may be in order. As shown in Figure 3, thyroidal $\mathrm{PB}^{131} \mathrm{I}$ increased progressively with time and constituted more than $80 \%$ of total thyroidal radioiodine at $24 \mathrm{hrs}$., while thyroidal radioiodine uptake remained low throughout $24 \mathrm{hrs}$. Since percentage of intrathyroidal organic iodine is governed by MTU but not by the reduction of circulating ${ }^{131} \mathrm{I}^{-}$ produced by the drug, our data suggest that thyroidal $\mathrm{PB}^{131} \mathrm{I}$ is more reliable index than thyroidal radioiodine uptake in MTU treated animals.

Since our purpose is to study which parameters of thyroid activity is the reliable index for measuring blocking action of antithyroid drug on thyroid hormone synthesis, and since thyroidal $\mathrm{PB}^{131} \mathrm{I}$ is comprised of hormones $\left(\mathrm{T}_{4}\right.$ and $\left.\mathrm{T}_{3}\right)$ and its precursor (MIT and DIT), we have further studied the reliability of $\mathrm{PB}^{131} \mathrm{I}$ as the criteria of thyroid hormone synthesis after a single injection of $20 \mathrm{mg}$

MTU. As shown in Figure 5, thyroidal $\mathrm{PB}^{131} \mathrm{I}$ only reflects MIT at $4 \mathrm{hrs}$. Even at $24 \mathrm{hrs}$., thyroidal $\mathrm{PB}^{131} \mathrm{I}$ well reflects MIT but not $\mathrm{T}_{4}$ and $\mathrm{T}_{3}$. Thus it can be concluded that blocking effect of antithyroid drug on thyroid hormone synthesis can only be evaluated by measuring thyroidal $T_{4}$ and $T_{3}$. However, measurement of intrathyroidal hormones is time consuming and not possible in in vivo experiment. In view of these reasons, we have further attempted to re-evaluate thyroidal radioiodine uptake and thyroidal $\mathrm{PB}^{131} \mathrm{I}$ as the criteria of thyroid hormone synthesis. In order to minimize a possible error produced by rapid disappearance of iodide, radioiodine was injected $4 \mathrm{hrs}$. before autopsy. Here we found that thyroidal uptake initially suppressed returned to normal by 4 days and exceeded normal value thereafter. A low value of thyroidal $\mathrm{PB}^{131} \mathrm{I}$ also returned to normal by 4 days. Furthermore, percentage of intra- 
thyroidal $T_{4}$ and $T_{3}$ returned to normal by 4 days. Thus, thyroidal radioiodine uptake and thyroidal $\mathrm{PB}^{131} \mathrm{I}$ well reflect synthesis of thyroid hormones when radioiodine is injected 4 hrs. before measurement. However, when judged by DIT/MIT ratio, the ratio was still abnormal at 4 days and normalized at 5 days, indicating some delay of this parameter.

Roche (Roche, 1951) have reported a decrease of thyroidal content of thyroglobulin when antithyroid drug was administered for a long period. The significance of this decrease remained unsolved. However, our present study clearly indicated that $20 \mathrm{mg}$ MTU actually depressed thyroidal content of thyroglobulin in which iodination of tyrosyl residue took place. This decrease seems not to be an accidental occurrence, since thyroglobulin content decreased when hormone synthesis is depressed by MTU and returned to normal when hormone synthesis is actually normal. Thus, it seems reasonable that antithyroid drug, at least MTU, acts on the thyroid in two ways; one blocking oxydative enzyme and the other depressing thyroglobulin content.

\section{Acknowledgement}

We are deeply indebted to Drs. Ui and Tarutani for the analysis of thyroidal protein by ultracentrifugation.

\section{References}

Brown, J. (1956). Endocrinology 58, 68.

Greer, M. A., J. W. Kendall and M. Smith, in Pitt-Rivers and Trotter (eds.), The Thyroid Gland Vol. 1, Butterworth, Washington, p.357 (1964).

Iino, S., T. Yamada and M. A. Greer (1961). Endocrinology 68, 582.

Maloof, F. and M. Soodak (1957). Ibid. 61, 555.

Richards, J. B. and S. H. Ingbar (1959). Ibid. 65, 198.

Roche, J., Quelques récentes acquisitions sur la biochimie de l'hormone thyroidienne, exposes annuels de biochimie medicale, 13th series, Masson, Paris, p.145 (1951).

Shimoda, S. and M. A. Greer (1966). Endocrinology 78, 715 .

Slingerland, D. W., D. E. Graham, R. K. Josephs, P. F. Mulvey, Jr., A. P. Trakas and E. Yamazaki (1959). Ibid. 65, 178.

Ui, N. and O. Tarutani (1961). J. Biochem. 50, 508. 